\title{
Louis FAVREAU
}

Sociologue, professeur au département de travail sociale de l’Université du Québec en Outaouais

Titulaire de la Chaire de recherche du Canada en développement des collectivités (CRDC)

Avec la collaboration de Sambou Ndiaye, Humberto Ortiz et Gérald Larose.

(2008)

\section{"Économie solidaire et coopération internationale”}

Un document produit en version numérique par Jean-Marie Tremblay, bénévole, professeur de sociologie au Cégep de Chicoutimi

Courriel: jean-marie tremblay@uqac.ca

Site web pédagogique : http://www.uqac.ca/jmt-sociologue/

Dans le cadre de: "Les classiques des sciences sociales" Une bibliothèque numérique fondée et dirigée par Jean-Marie Tremblay, professeur de sociologie au Cégep de Chicoutimi Site web: http://classiques.uqac.ca/

Une collection développée en collaboration avec la Bibliothèque Paul-Émile-Boulet de l'Université du Québec à Chicoutimi Site web: http://bibliotheque.uqac.ca/ 


\section{Politique d'utilisation de la bibliothèque des Classiques}

Toute reproduction et rediffusion de nos fichiers est interdite, même avec la mention de leur provenance, sans l'autorisation formelle, écrite, du fondateur des Classiques des sciences sociales, Jean-Marie Tremblay, sociologue.

Les fichiers des Classiques des sciences sociales ne peuvent sans autorisation formelle:

- être hébergés (en fichier ou page web, en totalité ou en partie) sur un serveur autre que celui des Classiques.

- servir de base de travail à un autre fichier modifié ensuite par tout autre moyen (couleur, police, mise en page, extraits, support, etc...),

Les fichiers (.html, .doc, .pdf., .rtf, .jpg, .gif) disponibles sur le site Les Classiques des sciences sociales sont la propriété des Classiques des sciences sociales, un organisme à but non lucratif composé exclusivement de bénévoles.

Ils sont disponibles pour une utilisation intellectuelle et personnelle et, en aucun cas, commerciale. Toute utilisation à des fins commerciales des fichiers sur ce site est strictement interdite et toute rediffusion est également strictement interdite.

L'accès à notre travail est libre et gratuit à tous les utilisateurs. C'est notre mission.

Jean-Marie Tremblay, sociologue

Fondateur et Président-directeur général, LES CLASSIQUES DES SCIENCES SOCIALES. 
Cette édition électronique a été réalisée par Jean-Marie Tremblay, bénévole, professeur de sociologie au Cégep de Chicoutimi à partir de :

\section{Louis Favreau}

Sociologue, professeur au département de travail sociale de l’Université du Québec en Outaouais, Titulaire de la Chaire de recherche du Canada en développement des collectivités (CRDC)

Avec la collaboration de Sambou Ndiaye, Humberto Ortiz et Gérald Larose.

\section{“Économie solidaire et coopération internationale.”}

Une série de deux articles publiés dans le journal Le Devoir, Montréal, éditions du mercredi 24 septembre 2008, page A7 et jeudi, 25 septembre 2008, page A9 -idées.

[Autorisation formelle accordée par l'auteur le 27 septembre 2008 de diffuser toutes ses publications dans Les Classiques des sciences sociales.]

Courriel : louis.favreau@uqo.ca

Polices de caractères utilisée :

Pour le texte: Times New Roman, 12 points.

Pour les citations : Times New Roman, 12 points.

Pour les notes de bas de page : Times New Roman, 12 points.

Édition électronique réalisée avec le traitement de textes Microsoft Word 2004 pour Macintosh.

Mise en page sur papier format : LETTRE (US letter), 8.5'’ x 11'’)

Édition numérique réalisée le 28 septembre 2008 à Chicoutimi, Ville de Saguenay, province de Québec, Canada.

\section{\& Fait avec}




\section{Louis FAVREAU}

Sociologue, professeur au département de travail sociale de l’Université du Québec en Outaouais Titulaire de la Chaire de recherche du Canada en développement des collectivités (CRDC)

Avec la collaboration de Sambou Ndiaye, Humberto Ortiz et Gérald Larose.

\section{"Économie solidaire et coopération internationale.”}
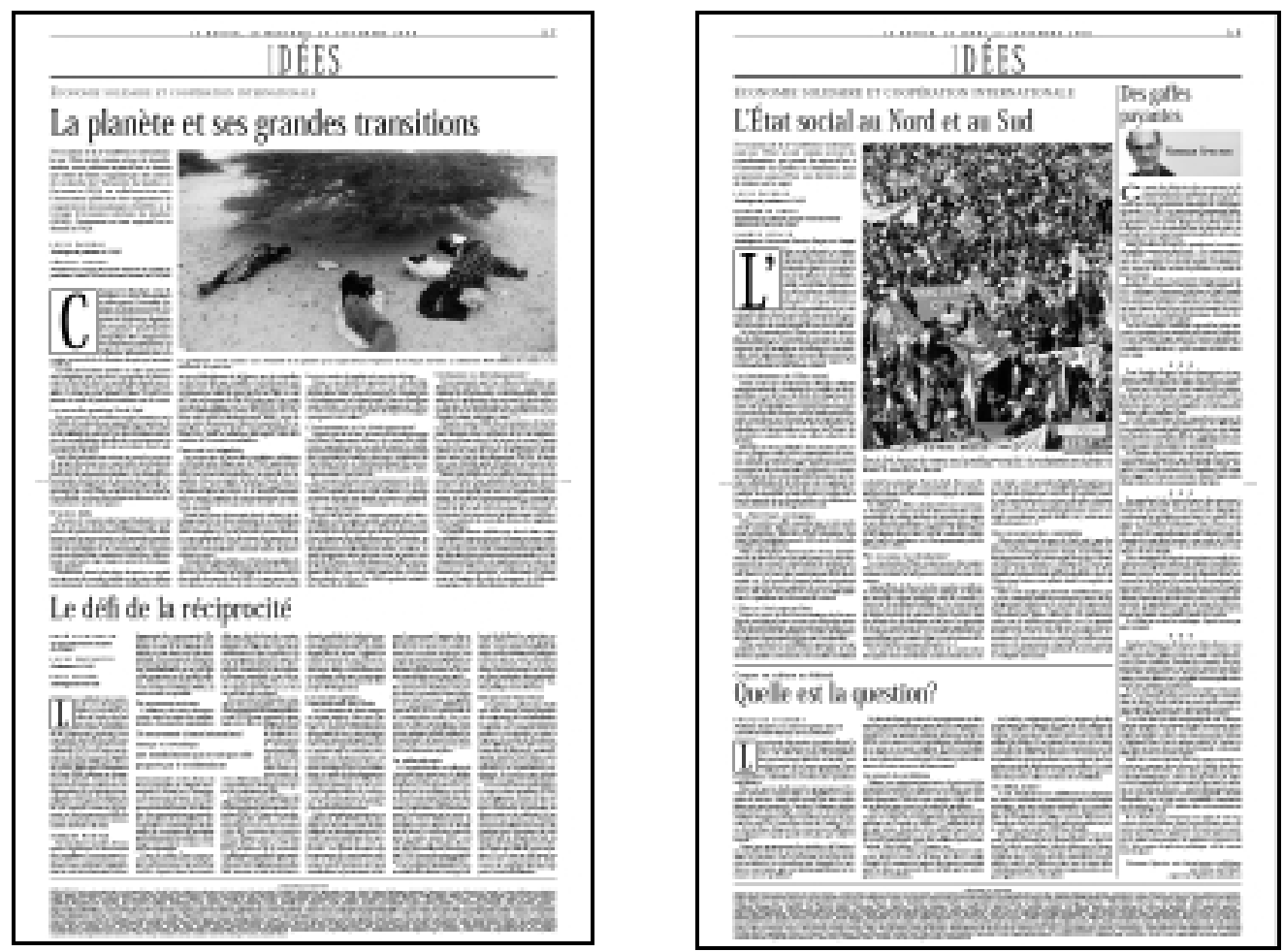

Une série de deux articles publiés dans le journal Le Devoir, Montréal, éditions du mercredi 24 septembre 2008, page A7 et jeudi, 25 septembre 2008, page A9 -idées. 


\section{Table des matières}

1. Louis Favreau et Gérald Larose, “La planète et ses qrandes transitions”. In Le Devoir, Montréal, éditions du mercredi 24 septembre 2008, page A7idées.

Gérald Larose, Président du Groupe d'économie solidaire du Québec et professeur invité à l'École de travail sociale de l'UQAM Louis Favreau, Sociologue et professeur à l'UQO

2. Louis Favreau, Sambou Ndiaje et Humberto Ortiz, “L'État social au Nord et au Sud”. In Le Devoir, Montréal, édition du jeudi, 25 septembre 2008, page A9 -idées.

Sambou Ndiaye, Sociologue à l'Université Gaston Berger au Sénégal

Humberto Ortiz, Économiste au GRESP (Grupo red de economía solidaria del Peru) du Pérou Louis Favreau, Sociologue et professeur à l'UQO 


\section{Louis FAVREAU}

Sociologue, professeur au département de travail sociale de l’Université du Québec en Outaouais

Titulaire de la Chaire de recherche du Canada en développement des collectivités (CRDC)

\section{“Économie solidaire et coopération internationale”.}

Une série de deux articles publiés dans le journal Le Devoir, Montréal, éditions du mercredi 24 septembre 2008, page A7 et jeudi, 25 septembre 2008, page A9 —idées.

À l’occasion de la 2e Conférence internationale sur l’État social comme acteur de transformation, nous publions aujourd'hui et demain une série de textes. Organisé par des centres de recherche de l’Université du Québec en Outaouais (UQO) en collaboration avec l'Association québécoise des organismes de coopération internationale (AQOCI) et le Groupe d'économie solidaire du Québec (GESQ), l'événement se tient aujourd'hui et demain à l’UQO. 
Gérald Larose, Président du Groupe d'économie solidaire du Québec et professeur invité à l'École de travail sociale de l'UQAM

Louis Favreau, Sociologue et professeur à l'UQO

Économie solidaire et coopération internationale 1.

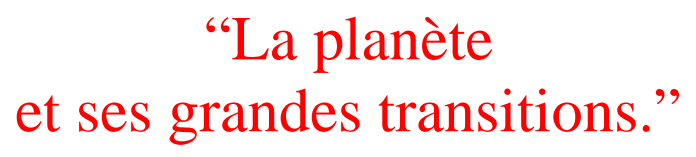

$\underline{\text { Retour à la table des matières }}$

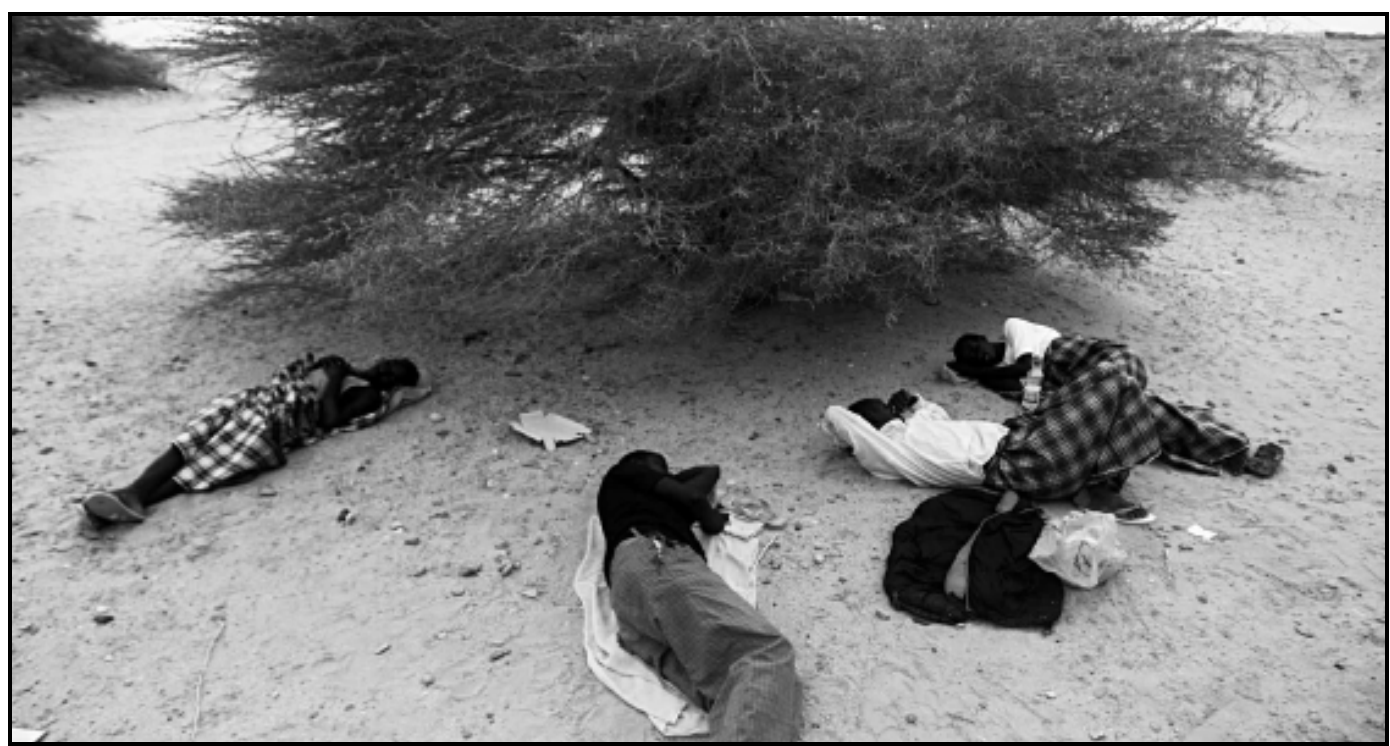

Texte de la photo : Le problème social numéro un à l'échelle de la planète peut aujourd'hui s'exprimer de la façon suivante: y subsistent 600 millions de riches et six milliards de pauvres. [Khaled Abdullah, Reuters.]

Mots clés : Coopération internationale, Économie solidaire, Aide à l'étranger, Économie, Canada (Pays), Québec (province)

À l'occasion de la 2e Conférence internationale sur l'État social comme acteur de transformation, nous publions aujourd'hui et demain une série de textes. Organisé par des centres de recherche de l'Université du Québec en Outaouais (UQO) en collaboration avec l'Association québécoise des organismes de coopération 
internationale (AQOCI) et le Groupe d'économie solidaire du Québec (GESQ), l'événement se tient aujourd'hui et demain à l'UQO.

Changement climatique, crise alimentaire et crise énergétique se télescopent. Désormais, certaines échéances peuvent être fatales étant donné les croisements de l'échéance climatique liée au seuil de réchauffement de la planète, de l'échéance énergétique liée à l'épuisement des ressources pétrolières (et sa gestion spéculative) et de l'échéance alimentaire liée à la remise au marché de la fixation des prix qui montent en flèche.

La toile de fond laisse poindre en outre une montée des inégalités qui consacre et perpétue la fracture entre le Nord et le Sud. Le monde dans lequel nous vivons est ainsi devenu plus instable et plus imprévisible. Ce n'est pas pour autant le chaos. Le pire n'est jamais sûr, mais de grandes transitions sont en cours.

\section{La nouvelle question Nord-Sud}

En raccourci, le problème social numéro un à l'échelle de la planète peut aujourd'hui s'exprimer de la façon suivante : y subsistent 600 millions de riches et six milliards de pauvres. Les deux dernières décennies de mondialisation ultralibérale ont notamment vu l'économie informelle devenir prévalente dans la plupart des pays du Sud.

Pour les mouvements sociaux et pour les porteurs de projets favorisant une coopération internationale de proximité, la recherche de formes plus appropriées de développement solidaire s'est imposée par la mutation du travail et de la protection sociale. Le travail salarié s'affaissant et l'économie informelle progressant, du coup, l'État social, là où il y en avait un, s'est affaibli et même littéralement dissous là où il n'était encore qu'en émergence.

\section{D'autres défis}

En outre, le constat selon lequel la planète est en état de survie sur le plan écologique remonte également à la surface dans tous les milieux. Plus nombreux sont les mouvements et de grandes organisations internationales qui remettent en ques- 
tion notre mode de production et de consommation. Comment peut-on aujourd'hui contribuer à faire face efficacement à cet autre enjeu majeur qu'est le développement durable?

Finalement, dans la dernière décennie, un troisième enjeu est devenu plus visible sur la scène publique internationale: la mondialisation ultralibérale a favorisé un tel débridement de la finance que les séquelles au Sud n'ont fait que s'amplifier. Les évidences sont nombreuses: du krach boursier au Mexique en 1994 en passant par celui des pays de l'Est en 1997 et celui de l'Asie au tournant de l'an 2000 alors que l'on venait tout juste de s'aligner sur les Objectifs du millénaire pour le développement (OMD, Programme des Nations unies pour le développement, 2003), sorte de pacte entre les pays pour vaincre la pauvreté. Et, aujourd'hui, on nage en pleine crise des liquidités aux États-Unis... Bref, un troisième défi majeur, celui de la maîtrise de l'économie mondialisée.

\section{Pauvreté et inégalités}

La perspective de nouvelles régulations politiques est d'autant plus obligée que les OMD apparaissent aujourd'hui dérisoires lorsque l'on redécouvre derrière cette pauvreté l'importance structurelle des inégalités. Deux exemples: le Mali et l'Inde. Dans le premier cas, deux millions de Maliens gagnent leur vie dans la filière du coton. Or, le marché international est sous l'emprise du coton américain et européen. Le Mali n'arrive pas à bien écouler son coton sur le marché.

Dans une jeune démocratie dont la volonté de développement et de démocratisation de ses institutions depuis près de 20 ans est particulièrement manifeste sinon exemplaire, c'est la précarité comme horizon. La cause principale ? Les structures du commerce international. Si on ne touche pas aux règles du jeu du commerce international, comment vaincre la pauvreté dans ce pays?

En Inde, les «intouchables» (25\% de la population de ce pays d'un milliard d'habitants) forment les «basses classes» d'une société qui, en dépit de sa démocratie, la plus vieille des pays du Sud (1947), n'a pas encore réussi à éliminer son

système de castes, lequel leur interdit l'accès à nombre d'emplois et de services de base. 
Dans ce cas, derrière la pauvreté, il y a la discrimination d'un système de castes. La question centrale devient donc: lutte contre la pauvreté ou combat pour le développement et la démocratie? Mais ne faut-il pas aussi revisiter cette notion de développement à l'heure où le consensus sur la gravité du risque écologique a progressé à vive allure ?

\section{L'humanitaire ou le développement?}

Depuis près de 50 ans, la notion de développement fait l'objet d'importants débats et de visions fort différentes, opposées même au sein de toutes les institutions internationales. Après l'échec fortement ressenti des coopérations étatiques des pays capitalistes du Nord dans le Sud, et celui démoralisant des mouvements de libération nationale et de leur modèle «développementiste», que reste-t-il ?

Le concept a été mis à mal et ramené par le FMI et la Banque mondiale à une simple gestion de la lutte contre l'extrême pauvreté. Pour d'autres, ce concept est même devenu une simple croyance occidentale. Face à ce relativisme économique ou culturel, un autre éclairage s'impose.

En près de 50 ans, nous sommes passés de la décolonisation, et des espoirs qu'elle suscitait alors, à une mondialisation ultralibérale. Ce début de basculement du monde n'a-t-il provoqué que du désenchantement ? Point culminant du désenchantement: l'abandon, en fait, du thème du développement dans les années 90, simultanément à l'effondrement du bloc socialiste. La seule perspective alors présente reste la montée des droits de l'Homme et, plus largement, l'effervescence de l'humanitaire devenu la quasi exclusive finalité légitime dans les rapports entre nations par ces temps d'incertitudes. Et avec l'an 2000 la grande initiative des Objectifs du millénaire.

\section{Croissance ou développement?}

Heureusement que l'on a fini par introduire une distinction majeure entre croissance, augmentation continue de la production, et développement, organisation de la production, des revenus et des dépenses en fonction de l'amélioration 
des conditions et de la qualité de vie de l'ensemble de la population d'un pays (emploi, habitat, éducation, santé...).

Dans les années 1990, des économistes du Sud introduisent un indice composite dont les trois principaux éléments sont l'espérance de vie à la naissance, le niveau d'instruction et le niveau de revenu. Depuis près de 20 ans, cet indice de développement humain (IDH) est le point de repère du Programme des Nations unies pour le développement (PNUD). Il donne lieu à une véritable petite révolution, car la porte de la pluridimensionnalité du développement a été ouverte.

Plutôt que d'infirmer la notion, plusieurs travaux avancent l'idée d'une pluralité des modèles dans le monde et de la pluridimensionnalité du développement, lequel prend son sens en combinant l'économique, le social et l'environnemental. Le concept est pertinent parce qu'il permet de réaffirmer la primauté de la société sur l'économie, de s'attaquer à la précarité, de promouvoir la démocratie et de favoriser la création de nouvelles formes de régulation sociopolitique.

La grande donnée oubliée reste donc la lutte contre les inégalités, perspective qui échappe presque totalement aux grandes institutions économiques internationales portées par le credo néolibéral et interpellées par des objectifs quasi exclusivement humanitaires. Le développement durable est également une notion qui peut s'avérer efficace dans le traitement des questions climatiques, énergétiques, ou alimentaires, notamment au chapitre des coûts de transport, de l'efficacité énergétique, de la délocalisation de la production. 
Sambou Ndiaye, Sociologue à l'Université Gaston Berger au Sénégal Humberto Ortiz, Économiste au GRESP (Grupo red de economía solidaria del Peru) du Pérou

Louis Favreau, Sociologue et professeur à l'UQO

Économie solidaire et coopération internationale 2.

$$
\text { "L'État social au Nord et au Sud." }
$$

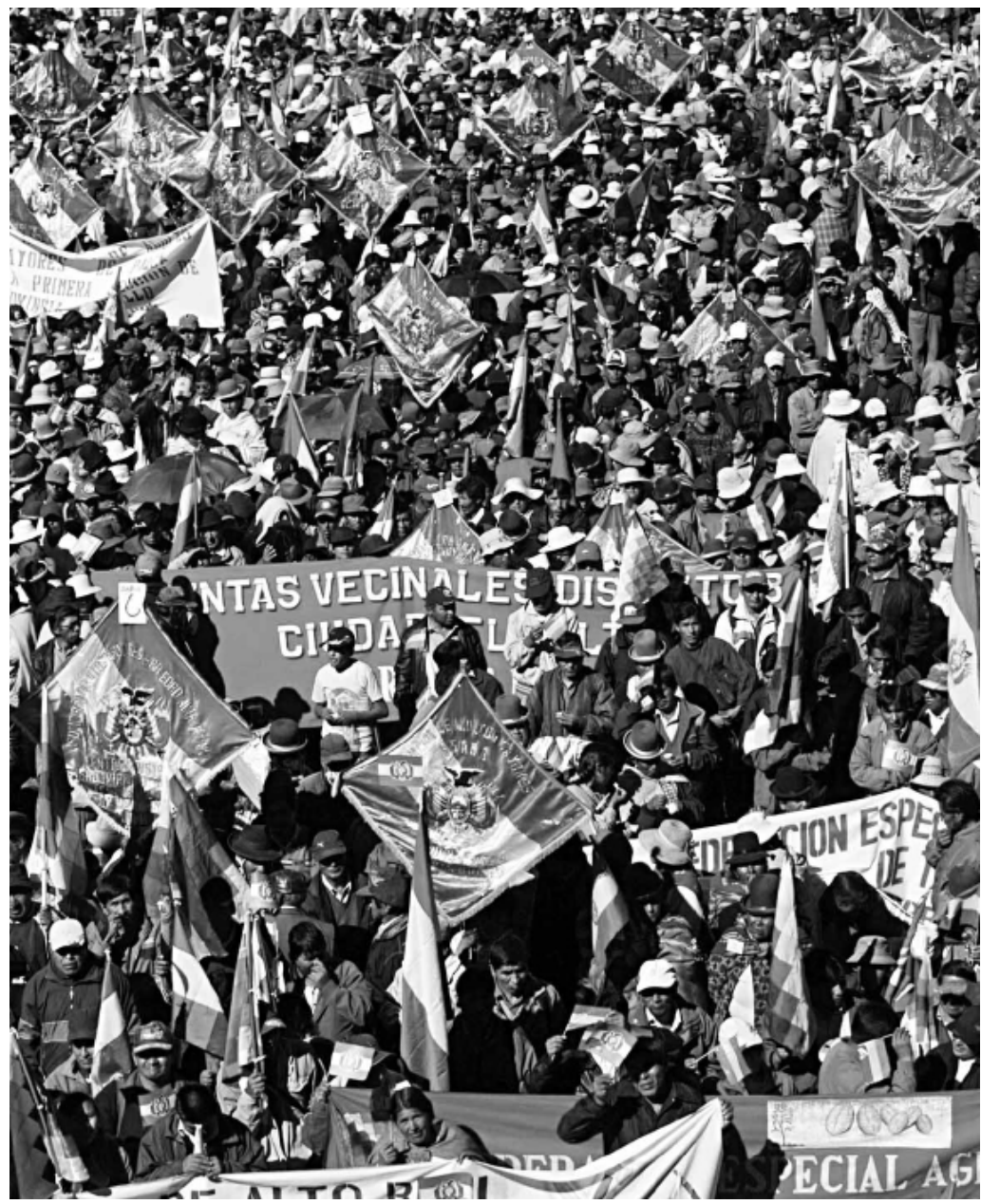

Texte de la photo : Pour le Sud, force est de rompre avec le modèle extraverti lié à la recolonisation par la dette et à l’arrimage à la logique du «tout au marché mondial». Ci-dessus, une manifestation proMorales, en Bolivie au mois de mai dernier. [Pilar Olivares, Reuters.]

Mots clés : Coopération internationale, Économie solidaire, État social, Gouvernement, Économie, Québec (province) 
À l'occasion de la 2e Conférence internationale sur l'État social comme acteur de transformation, qui prend fin aujourd'hui à l'Université du Québec en Outaouais, nous proposons aujourd'hui une dernière série de textes sur le sujet.

État social, adossé à un régime démocratique dans les sociétés du Nord et parachevé à la fin de la dernière guerre, s'est appuyé sur trois piliers: le développement d'un important service public, une fiscalité redistributive de la richesse collective et une action sur l'économie et l'emploi. Cet État social au Nord est partiellement en crise puisqu'il a laissé dans la précarité une partie de sa population active et exclut les usagers et les travailleurs des processus de construction des services collectifs.

Au Sud, la question de l'État social suscite encore plus de débats, car l'économie de ces pays a été profondément fragilisée par les changements structurels imposés par les institutions économiques internationales. Leur régime politique est aussi plus souvent autoritaire que démocratique. Par où passe aujourd'hui la reconstruction au Sud d'États sociaux ?

\section{Les fondements de l'État social}

L'État social a des fondements collectifs : solidarité intergénérationelle, résolutions des conflits par la négociation et sur la base du droit, développement de services publics dans les communautés, etc. Comment cela se traduit-il? Par le rapport impôt-citoyenneté, par une fiscalité obtenue sur un financement librement consenti par ses citoyens, par un impôt qui est le prix que nous payons pour avoir des enseignants, des infirmières et des travailleurs sociaux, des routes, de l'électricité à moindre coût, une collecte sélective des déchets...

Bref des services collectifs, dont certains sont publics et d'autres confiés à des organisations de caractère collectif (coopératives et associations) recevant une mission de service public. Expression de la citoyenneté et de la démocratie, l'argent de ces impôts sert à financer des services publics favorisant une certaine redistribution de la richesse tout en agissant sur l'économie et l'emploi. Bref, dans une société démocratique, la demande sociale favorise la conformité à l'intérêt 
général de ses fonds publics et la délibération sur les priorités de développement social.

\section{Un «New Deal» écologique}

L'État social ne suffit cependant pas à cette seule échelle nationale. Il faut aussi agir globalement. Dans cette perspective, l'idée des Verts européens d'un «New Deal écologique» pour le XXIe siècle à l'échelle mondiale est pertinente.

Mais cela implique beaucoup de choses: l'annulation de la dette des pays les plus pauvres, le développement d'un commerce international de produits écologiquement sains dont le commerce équitable est une amorce, la protection internationale de la biodiversité, etc. Un travail de longue haleine nécessitant qu'une majorité de pays agissent de concert. Une utopie ? Le XXe siècle n'a-t-il pas vu surgir un «New Deal» entre le capitalisme et le mouvement ouvrier?

\section{L'État au Sud aujourd'hui}

Dans les pays du Sud, les institutions de Bretton Woods ont imposé leurs recettes néo-libérales: moins d'État, des privatisations, une pression par la dette, le refus de financement de politiques sociales, la limitation de la liberté d'associations; la précarité s'est installée.

L'État a perdu de la crédibilité, la pauvreté s'est généralisée. Avec les récentes crises de la faim, le seuil de l'inacceptable a été franchi, et le modèle néo-libéral a atteint la saturation. Pour le Sud, force est de rompre avec le modèle extraverti lié à la recolonisation par la dette et à l'arrimage à la logique du «tout au marché mondial».

En Afrique, où l'État a d'abord été une excroissance étrangère, le processus de démocratisation a souvent risqué de rendre les lieux de pouvoir aux «clans» et aux minorités politiques. En Amérique latine, l'État a largement entretenu les privilèges et le clientélisme. À des degrés divers donc, sur un continent comme dans l'autre, la tendance est à l'accaparement privé de l'État par des élites. 


\section{Par où passe la refondation?}

La construction d'États sociaux passe donc aujourd'hui en bonne partie par les communautés, les mouvements sociaux et des gouvernements locaux innovateurs.

Aujourd'hui, de façon de plus en plus manifeste, en Amérique latine mais aussi en Afrique, se dessine une nouvelle volonté politique, celle du contrôle citoyen sur les politiques publiques. Les mouvements sociaux ont aussi très souvent dépassé aujourd'hui le simple pouvoir de nuisance pour s'inscrire dans des démarches de dialogue social avec les autorités locales. De nombreux acteurs sociaux appellent à la reconfiguration de l'État. Le modèle de développement misant sur le «tout à l'État» et celui misant sur le «tout au marché» n'ont pas débouché. [...]

Une partie de l'innovation dans la construction émergente d'un nouvel État social au Sud nous renvoie ainsi à cette première famille d'expériences (conseils de quartier ou «budgets participatifs») où l'on cherche avant tout à exercer un certain contrôle sur la production de services collectifs, réduisant ainsi les espaces de corruption dans des sociétés où les processus d'institutionnalisation (avec ses règles, procédures, appels d'offre, etc.) sont encore embryonnaires. [...]

\section{Une reconstruction contrastée}

L'État social dans les pays du Sud, mis à part les pays émergents et assez fortement industrialisés, demeure encore largement une utopie. L'asymétrie du développement et de la démocratie d'un pays à l'autre est fortement contrastée. L'Afrique de l'Ouest, par exemple, évolue dans les wagons de queue du développement humain tandis que la majorité des pays de l'Amérique latine sont plutôt dans les wagons du centre. [...]

Mais cette utopie peut devenir mobilisatrice grâce à un ensemble inédit de nouveaux acteurs de développement luttant pour la création d'espaces politiques démocratiques. Ces acteurs nouveaux auront cependant leur pleine efficacité s'ils établissent entre eux de solides passerelles avec les grands mouvements qui oeuvrent déjà sur la scène internationale et qui sont en voie de se reconfigurer, no- 
tamment le mouvement syndical, le mouvement paysan et le mouvement coopératif, le tout soutenu par une coopération internationale de proximité qui se redéploie autrement.

\section{Fin du texte}

